\title{
LAMP-Seq enables sensitive, multiplexed COVID-19 diagnostics using molecular barcoding
}

\author{
Kerstin U. Ludwig1, Ricarda M. Schmithausen $\mathbb{1}^{2}$, David Li3,4,5,6, Max L. Jacobs ${ }^{17,8}$, Ronja Hollstein', \\ Katja Blumenstock7, Jana Liebing ${ }^{9}$, Mikołaj Słabicki ${ }^{3,10,11}$, Amir Ben-Shmuel' ${ }^{12}$, Ofir Israeli' ${ }^{13}$, \\ Shay Weiss ${ }^{12}$, Thomas S. Ebert ${ }^{7}$, Nir Paran ${ }^{12}{ }^{12}$, Wibke Rüdiger ${ }^{7}$, Gero Wilbring ${ }^{\circledR 2}{ }^{2}$, David Feldman ${ }^{14}$, \\ Bärbel Lippke1, Nina Ishorst ${ }^{1,15}$, Lara M. Hochfeld ${ }^{1}{ }^{1}$, Eva C. Beins ${ }^{11}{ }^{1}$, Ines H. Kaltheuner ${ }^{16}$, \\ Maximilian Schmitz ${ }^{16}$, Aliona Wöhler ${ }^{17}$, Manuel Döhla ${ }^{\circledR 2,18}{ }^{2}$, Esther Sib ${ }^{2}$, Marius Jentzsch7, \\ Eva-Maria C. Moench ${ }^{19}$, Jacob D. Borrajo ${ }^{3,6}$, Jonathan Strecker ${ }^{3,4,5,6}$, Julia Reinhardt ${ }^{9}$, Brian Cleary $\mathbb{1}^{3}$, \\ Matthias Geyer ${ }^{16}$, Michael Hölzel ${ }^{9}$, Rhiannon Macrae ${ }^{1}{ }^{3,4,5,6}$, Markus M. Nöthen', Per Hoffmann ${ }^{1,20}$, \\ Martin Exner ${ }^{2}$, Aviv Regev $\mathbb{1}^{3,21,22,23,24,25}$, Feng Zhang $\mathbb{1}^{3,4,5,6,24}$ and Jonathan L. Schmid-Burgk $\mathbb{1}^{3,4,5,6,7 凶}$
}

Frequent testing of large population groups combined with contact tracing and isolation measures will be crucial for containing Coronavirus Disease 2019 outbreaks. Here we present LAMP-Seq, a modified, highly scalable reverse transcription loop-mediated isothermal amplification (RT-LAMP) method. Unpurified biosamples are barcoded and amplified in a single heat step, and pooled products are analyzed en masse by sequencing. Using commercial reagents, LAMP-Seq has a limit of detection of $\mathbf{2 . 2}$ molecules per $\mu \mathrm{l}$ at $\mathbf{9 5 \%}$ confidence and near-perfect specificity for severe acute respiratory syndrome coronavirus 2 given its sequence readout. Clinical validation of an open-source protocol with 676 swab samples, 98 of which were deemed positive by standard RT-qPCR, demonstrated $100 \%$ sensitivity in individuals with cycle threshold values of up to 33 and a specificity of $\mathbf{9 9 . 7 \%}$, at a very low material cost. With a time-to-result of fewer than $\mathbf{2 4}$ h, low cost and little new infrastructure requirement, LAMP-Seq can be readily deployed for frequent testing as part of an integrated public health surveillance program.

A of February 2021, the global spread of a novel coronavirus, severe acute respiratory syndrome coronavirus 2 (SARS-CoV-2), has resulted in over 109 million confirmed cases, and approximately 2.4 million deaths have been attributed to Coronavirus Disease 2019 (COVID-19)1․ Current containment strategies based on 'test-trace-isolate' face major issues: (1) many infected individuals do not show any symptoms and, therefore, remain untested ${ }^{2}$; (2) supply chain issues limit testing capacity; and (3) the successive (rather than parallel) testing of contact individuals causes a substantial lag in identifying infection chains, resulting in undetected spread due to delayed diagnosis. By contrast, repeated testing of large groups of individuals, regardless of symptoms or contact status, is predicted to be an effective measure to decrease SARS-CoV-2 transmission ${ }^{3-5}$. Furthermore, this strategy helps to pinpoint outbreak areas and ongoing community transmission, thus enabling local interventions that maximize human health while minimizing the societal impact of restrictive isolation measures.

The current gold standard diagnostic test for detection of active SARS-CoV-2 infection is viral RNA extraction from a biospecimen followed by RT-qPCR to amplify and detect conserved regions of the SARS-CoV-2 genome. With increasing infection numbers, this has been complemented by antigen-based tests, which provide rapid results but have limited sensitivity ${ }^{6}$, and sequencing-based approaches, which have increased throughput but still require

'Institute of Human Genetics, University of Bonn and University Hospital Bonn, Bonn, Germany. ${ }^{2}$ Institute of Hygiene and Public Health, University of Bonn and University Hospital Bonn, Bonn, Germany. ${ }^{3}$ Broad Institute of MIT and Harvard, Massachusetts Institute of Technology, Cambridge, MA, USA. ${ }^{4} \mathrm{McGovern}$ Institute for Brain Research, Massachusetts Institute of Technology, Cambridge, MA, USA. ${ }^{5}$ Department of Brain and Cognitive Sciences, Massachusetts Institute of Technology, Cambridge, MA, USA. ${ }^{6}$ Department of Biological Engineering, Massachusetts Institute of Technology, Cambridge, MA, USA. ${ }^{7}$ Institute of Clinical Chemistry and Clinical Pharmacology, University of Bonn and University Hospital Bonn, Bonn, Germany. ${ }^{8}$ Center for Molecular Biology of Heidelberg University (ZMBH), Heidelberg, Germany. ${ }^{9}$ Institute of Experimental Oncology, University of Bonn and University Hospital Bonn, Bonn, Germany. ${ }^{10}$ Department of Medical Oncology, Dana-Farber Cancer Institute, Boston, MA, USA. "Division of Translational Medical Oncology, German Cancer Research Center (DKFZ) and National Center for Tumor Diseases (NCT), Heidelberg, Germany. ${ }^{12}$ Department of Infectious Diseases, Israel Institute for Biological Research, Ness Ziona, Israel. ${ }^{13}$ Department of Biochemistry and Molecular Genetics, Israel Institute for Biological Research, Ness Ziona, Israel. ${ }^{14}$ Department of Biochemistry and Institute for Protein Design, University of Washington, Seattle, WA, USA. ${ }^{15}$ Institute of Anatomy, Division of Neuroanatomy, University of Bonn and University Hospital Bonn, Bonn, Germany. ${ }^{16}$ Institute of Structural Biology, University of Bonn and University Hospital Bonn, Bonn, Germany. ${ }^{17}$ Department of General, Visceral and Thoracic Surgery, Bundeswehr Central Hospital Koblenz, Koblenz, Germany. ${ }^{18}$ Department of Microbiology and Hospital Hygiene, Bundeswehr Central Hospital Koblenz, Koblenz, Germany. ${ }^{19}$ MVZ Laboratory, Dr. Quade and Associates, Cologne, Germany. ${ }^{20} \mathrm{Genomics}$ Research Group, Department of Biomedicine, University of Basel, Basel, Switzerland. ${ }^{21}$ Department of Biology, Massachusetts Institute of Technology, Cambridge, MA, USA. ${ }^{22}$ Klarman Cell Observatory, Massachusetts Institute of Technology, Cambridge, MA, USA. ${ }^{23}$ Koch Institute for Integrative Cancer Research, Massachusetts Institute of Technology, Cambridge, MA, USA. ${ }^{24} \mathrm{Howard} H u g h e s$ Medical Institute, Cambridge, MA, USA. ${ }^{25}$ Present address: Genentech, South San Francisco, CA, USA. $\varpi_{e}$-mail: jonathan.schmid-burgk@ukbonn.de 
RNA extraction and/or thermocycling devices ${ }^{7-11}$. Here we describe LAMP-Seq, an approach that combines RT-LAMP ${ }^{12,13}$ with molecular barcoding to detect viral genomes in unpurified lysates at high throughput.

\section{Results}

Scalable deep sequencing-based SARS-CoV-2 detection. RTLAMP uses six target-specific primers and a strand-displacing polymerase (Fig. 1a), and it has been shown to detect pathogens in unpurified samples at high sensitivity ${ }^{14}$. To establish a barcoded RT-LAMP reaction suitable for large-scale next-generation sequencing (NGS)-based detection, we designed a barcoded primer set based on a validated RT-LAMP amplicon ${ }^{15}$ that matched $94.1 \%$ of 42,904 SARS-CoV-2 genomes available in the National Center of Biotechnology Information database (as of 11 March 2021; Supplementary Data 1). The barcodes (10-nt long, GC content of $30-70 \%$ and lacking homopolymer repeats of four or more nucleotides) were inserted into the forward inner primer (FIP) (denoted as 'LAMP barcodes' (LAMP-BCs)) (Fig. 1b). To reduce the risk of sample misattribution due to sequencing errors, we ensured a Levenshtein edit distance ${ }^{16}$ between any barcode pair of three or more. Further scalability can be achieved by introducing a second level of barcodes at the PCR stage on pooled samples, using two standard PCR barcodes (PCR-BCs): i5 and i7 (Supplementary Data 1). The final structure of the sequencing library is shown in Fig. $1 b$.

We first determined the molecular sensitivity of barcoded RTLAMP reactions using in vitro transcribed (IVT) template RNA and commercial RT-LAMP reagents (Methods). We included F3 and B3 primers with or without locked nucleic acid (LNA) modifications, which can increase binding affinity ${ }^{17}$ (Fig. 1c; positions of LNA modifications are described in Methods). We obtained a limit of detection at 95\% confidence (LoD-95) of about 18 RNA molecules per assay, using probit analysis (equivalent to 2.2 copies per $\mu \mathrm{l}$ (Fig. 1d)). This is similar to the assay sensitivity of the standard $E$ gene RT-qPCR assay used for clinical diagnostics at the University Hospital Bonn (LoD-95: 18.4 molecules, using IVT RNA). Templating individually barcoded LAMP reactions that differ 100- or 10,000 -fold in the amount of RNA template, combining them for PCR amplification and sequencing the products resulted in saturated read numbers (Fig. 1e). This indicates that RT-LAMP saturation effectively compresses the dynamic range from input viral loads to sequencing reads, enabling analysis of large numbers of samples spanning a wide range of viral loads in one sequencing run.

Next, we optimized the pooling of barcoded RT-LAMP reactions to (1) minimize the levels of barcode swapping and (2) ensure a sufficient number of individually validated barcodes. We observed moderate levels of barcode swapping when we pooled six barcoded RT-LAMP reactions, three of which were templated with IVT RNA before PCR and sequencing (Fig. 1f, left panel). We hypothesized that introducing barcoded primers into the PCR reaction led to amplification and re-barcoding of amplicons. In support of this, we eliminated detectable barcode swapping by diluting the pooled RT-LAMP reactions $10^{6}$-fold in the final PCR reaction (Fig. 1f, right panel). Finally, we pooled 480 barcoded FIPs and performed LAMP-Seq in quadruplicate. The barcode distribution in the products revealed that $\sim 5 \%$ of barcode sequences performed poorly or even failed to engage in LAMP-Seq (Fig. 1g). The least efficient barcode primers displayed a marked enrichment for a GTCC motif or truncations thereof, especially toward the $3^{\prime}$ end of the barcode (Fig. 1g, inset). This is the reverse complement of the $3^{\prime}$ end of the FIP, suggesting that it was sequestering FIPs at their $3^{\prime}$ end by forming intramolecular structures. Thus, this homology should be avoided in barcode design, and we provide 192 experimentally validated FIP barcodes in Supplementary Data 1 (N-FIP barcode primers TIER-1). To enable scaling of LAMP-Seq, we additionally tested an $\mathrm{N}_{10}$-barcode library and identified about 10,000 functional barcodes. Of these, we in silico curated a set of 3,840 barcodes based on a minimum 3-edit distance, which are independent from Primer Set TIER 1 (Supplementary Data 1 (N-FIP barcode primers TIER-2)).

For LAMP-Seq to be safely deployed on patient samples, we confirmed that SARS-CoV-2 virus was inactivated in QuickExtract lysis buffer both after $30 \mathrm{~min}$ of incubation at $65^{\circ} \mathrm{C}$ and after $10 \mathrm{~min}$ at $95^{\circ} \mathrm{C}$. Both incubations resulted in a $>40,000$-fold reduction in viral infectivity, whereas a 30 -min incubation at $22^{\circ} \mathrm{C}$ resulted in residual SARS-CoV-2 infectivity. The inactivation efficiency of the lysis buffer was further demonstrated by observing a $>10^{7}$-fold reduction in viral infectivity of high dose of vesicular stomatitis virus (VSV) after incubation at $65^{\circ} \mathrm{C}$ for $20 \mathrm{~min}$ (Supplementary Table 1).

Clinical validation of LAMP-Seq using commercial reagents. We next tested 57 high-incidence human samples using LAMP-Seq side by side with a clinically approved diagnostic RT-qPCR pipeline. Upon informed consent, two oropharyngeal swab samples were collected from each individual using two separate cotton swabs. One randomly selected swab was analyzed using a validated clinical diagnostics pipeline comprising rehydration, robotic RNA purification and RT-qPCR using $E$ gene-specific primers (which have been reported to be more sensitive than primers for other targets ${ }^{18}$; Fig. 2a, upper panel). The other swab was immediately inserted into a tube containing QuickExtract lysis buffer ${ }^{19}$ (Fig. 2a, lower panel), and $8.3 \mu$ of lysate was processed with LAMP-Seq in quadruplicate, using individual LAMP-BCs and PCR-BCs, with a multiplexed E1 or $\beta$-actin control in one of the replicates (Methods). Unfiltered LAMP-Seq data displayed the expected read structure, comprising primer sequences, viral genome sequences and matching barcodes (Fig. 2b). After sequencing, the median read count for four positive replicates was determined, and sample replicates were deemed positive if they showed at least $10 \%$ of that read number. Twenty-two of 25 individuals who were identified as positive for SARS-CoV-2 RNA by RT-qPCR were also identified as positive by LAMP-Seq (with two or more positive replicates). The three non-detected samples showed very low viral titers in the clinical pipeline (cycle threshold (Ct): 36.96-38.52). The remaining 32 individuals were identified as negative for viral RNA (with above-threshold number reads for $\beta$-actin; Fig. 2c). Together, our data using commercial LAMP reagents suggest that LAMP-Seq is a highly sensitive and specific SARS-CoV-2 testing approach.

Development of an open-source LAMP-Seq protocol. To further increase the potential for large-scale application of LAMP-Seq, we established an open-source version that allows for substantial cost reduction and independence from supply chains. We replaced all cost-driving items (lysis buffer and LAMP enzyme master mix) by self-produced buffers and enzymes (Methods) and reduced dNTP and primer concentrations. Notably, we found that a Bst polymerase large fragment from a Geobacillus strain sampled in Idaho ${ }^{20}$ (Bst-LF-Idaho) alone is sufficient to maintain a high sensitivity of LAMP-Seq, without a reverse transcriptase but in the presence of LNA modifications in the F3/B3 primers (Supplementary Fig. 1). Applying this open-source protocol to different amounts of chemically inactivated SARS-CoV-2 particles (Methods) revealed slightly increased LoD-95 of 39 molecules per reaction or about 4.7 molecules per $\mu$ (Fig. 3a). We also confirmed high LAMP-Seq specificity, as we did not detect any of 15 other viral sequences (Fig. 3b). As cross-contamination of adjacent wells is a major concern in LAMP protocols, we performed a checkerboard experiment with 192 positive and 192 negative samples in duplicates (Fig. 3c). Using LAMP-BC Primer Set TIER 1, we observed a robust black-white pattern as expected. Sporadic sub-threshold reads (less than $10 \%$ of median positive reads) occurred in seven of 192 positions, and we also observed 

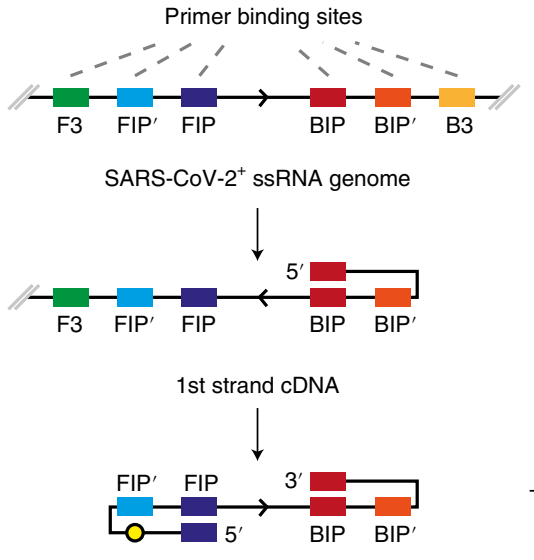

2nd strand cDNA

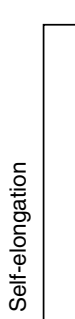$$
3^{\prime}
$$
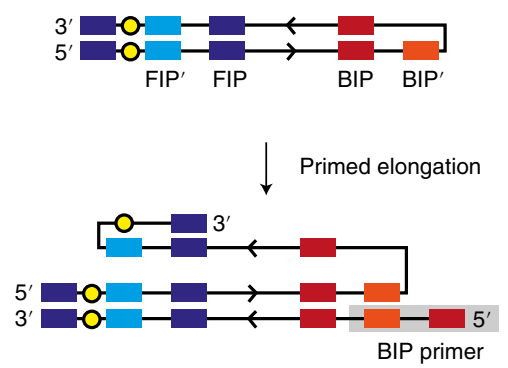

Self-elongation
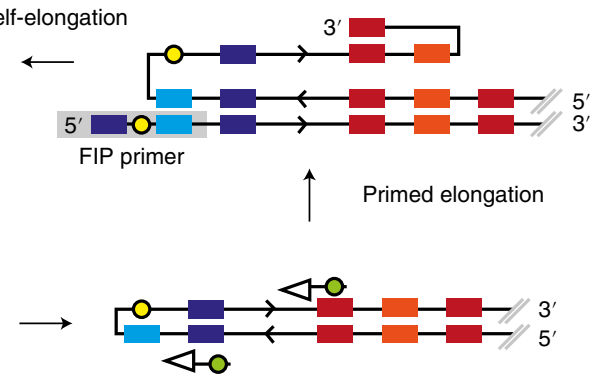

$\downarrow$ PCR

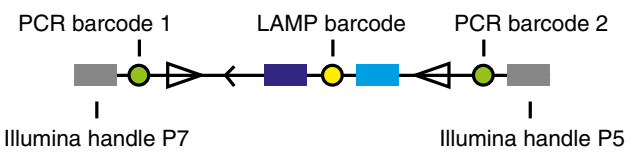

b

Illumina handle $\mathrm{P} 7$

PCR barcode 1

Sequencing primer binding site

PCR forward primer

AATGATACGGCGACCACCGAGATCTACACTCGTGGAGCGACACTCTTTCCCTACACGACGCTCTTCCGATCTAACGCTGAAGCGCTGGGGGCAAA ..

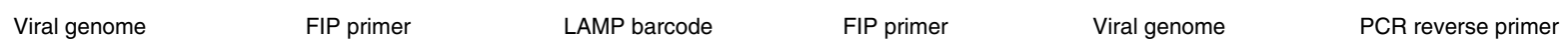
TTGTGCAATTTGCGGCCAATGTTTGTAATCAGAGCTTACACTCCAAGGAAATTTTGGGGACCAGGAACTAATCAGACAAGGAACTGATTACAAACAG ...

\section{Sequencing primer binding site \\ PCR barcode 2 \\ Illumina handle P5 \\ ATCGGAAGAGCACACGTCTGAACTCCAGTCACGAACTGAGCGATCTCGTATGCCGTCTTCTGCTTG}

C

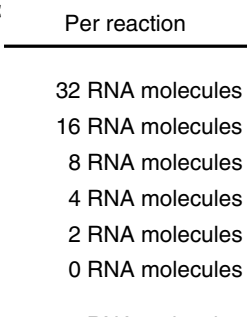

8 RNA molecules

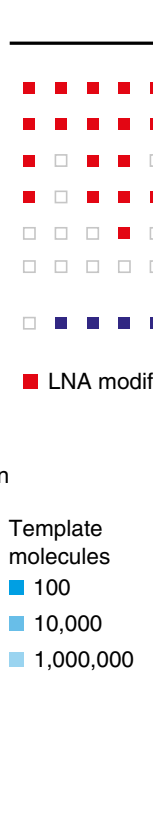

24 independent LAMP-seq reactions

\begin{tabular}{c} 
Positive \\
\hline $24 / 24$ \\
$21 / 24$ \\
$18 / 24$ \\
$13 / 24$ \\
$9 / 24$ \\
$0 / 24$ \\
$7 / 24$
\end{tabular}

no modification

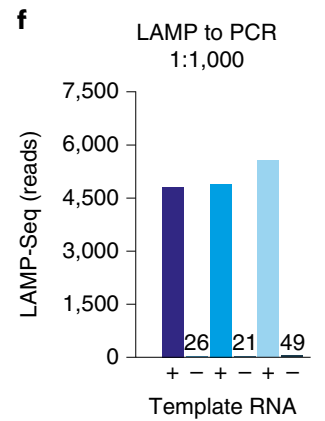

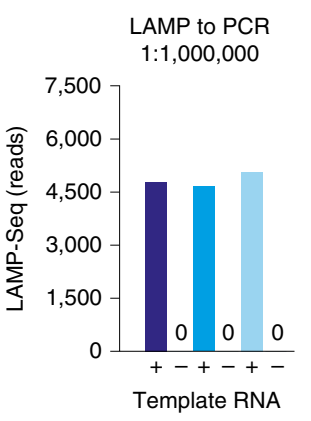

d

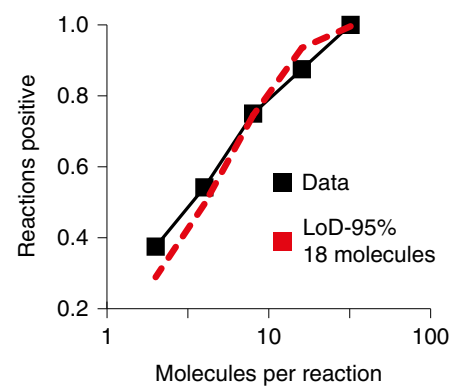

g

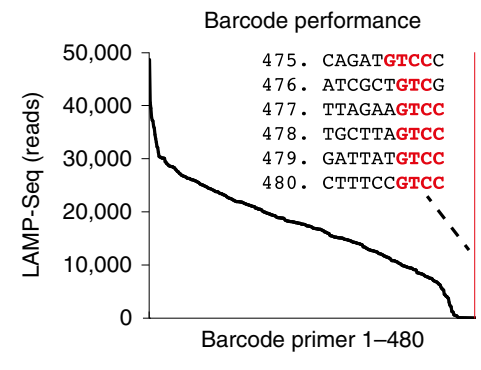

Fig. 1 | LAMP-Seq: a scalable deep sequencing-based approach for SARS-CoV-2 detection. a, Schematic of anticipated enzymatic reactions and reaction products. b. Annotated amplicon sequence for Illumina NGS of SARS-CoV-2 RT-LAMP products. c, Sensitivity assessment of LAMP-Seq using 24 reactions per condition, templated with the indicated numbers of RNA molecules per reaction. Filled squares indicate barcodes with read numbers $>10 \%$ of median calculated from 24 positive samples. LNA modifications increase melting temperature from $53.7^{\circ} \mathrm{C}$ to $60.1^{\circ} \mathrm{C}$ for $\mathrm{F} 3$ and from $50.0^{\circ} \mathrm{C}$ to $57.3^{\circ} \mathrm{C}$ for B3. d, Estimation of the LoD-95 based on probit analysis of the fraction of positives among 24 replicates. e, LAMP-Seq reactions templated with $100,10,000$ or 100,000 RNA molecules. Reactions were PCR amplified and sequenced on an Illumina MiSeq sequencer. Absolute read counts per sample are shown from ten experimental replicates per condition. $\mathbf{f}$, Quantitative assessment of barcode swapping in LAMP-Seq and dependence on pre-dilution of pooled RT-LAMP reactions before PCR (left panel, 1,000-fold; right panel, 1,000,000-fold). LAMP-Seq was performed as described in the Methods section, with the exception that synthetic RNA was used as the template instead of a swab sample, no Bst 3.0 or Tris buffer was added, and no LNA-modified primers were used. Numbers in the plot indicate read numbers for non-templated negative control reactions. $\mathbf{g}$, Empirical performance assessment of 480 randomly chosen LAMP-Seq barcode primers. The barcoded FIPs were mixed at an equimolar concentration and used as a pool in four replicate LAMP-Seq reactions templated by RNA. Raw sequencing data were analyzed using LAMP-Seq Inspector v1.0 (http://manuscript.lamp-seq. $\mathrm{org} /$ Inspector.htm). Read counts are shown for barcodes in descending order. The six worst-performing barcode sequences are highlighted in red, and the respective sequences are provided in the inset. ssRNA, single-stranded RNA. 


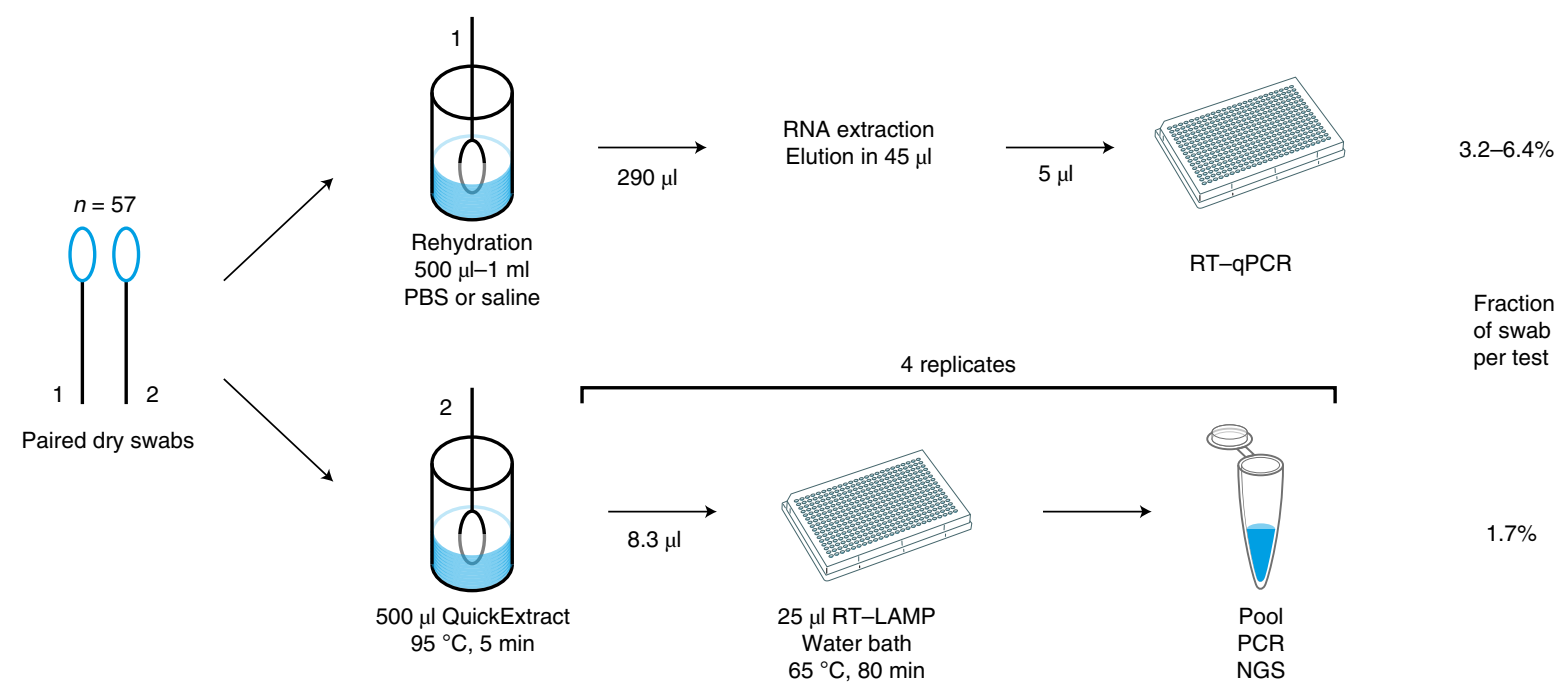

b

Unfiltered sequencing data from positive swab

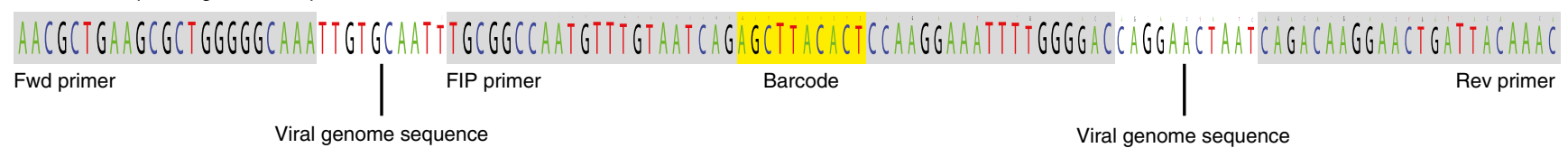

C
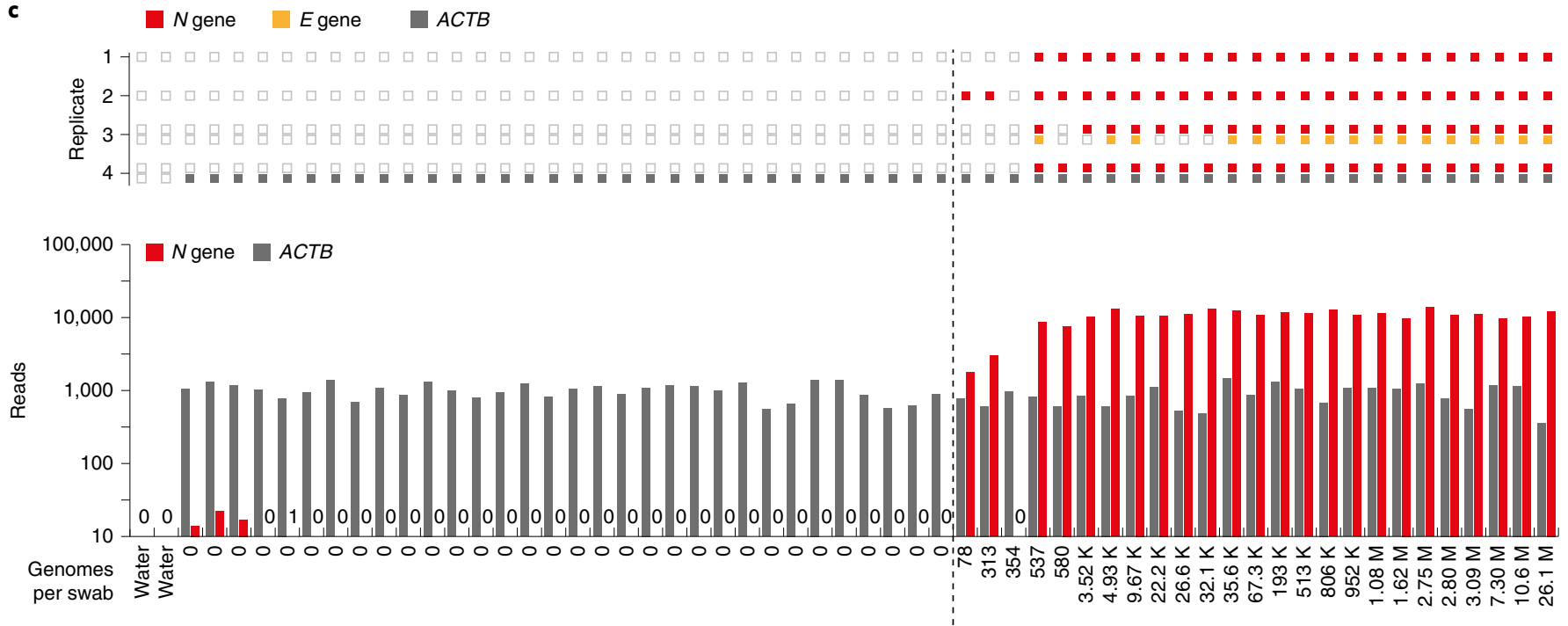

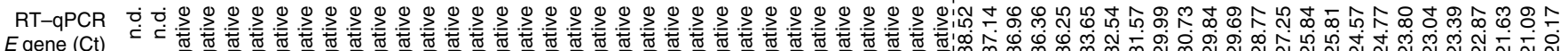

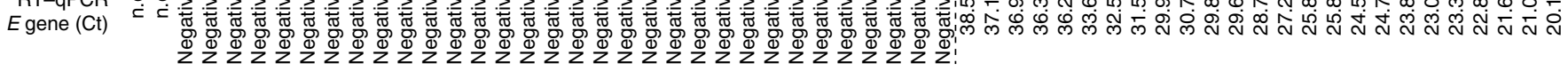

Fig. 2 | Clinical validation of LAMP-Seq. a, Outline of the protocol employed for validating LAMP-Seq (bottom workflow) against an established clinical RT-qPCR pipeline (top workflow). b, Sequencing data obtained from a SARS-CoV-2-positive swab sample using LAMP-Seq. Base frequencies are depicted by the size of each letter without applying any read filtering. c, Upper panel, positive LAMP-Seq replicates (threshold at $10 \%$ of median read number of four LAMP-Seq reactions with lowest corresponding Ct values) are indicated by filled squares. Replicates 1 and 2 detect just the $N$ gene; replicate 3 is multiplex detection for $N$ and $E$ genes; replicate 4 is multiplex detection for $N$ gene and ACTB ( $\beta$-actin; endogenous control). Lower panel, cumulative read numbers obtained per sample, ordered by estimated viral genomes per swab as determined by RT-qPCR (E gene) on a paired swab, taking into account different rehydration volumes. The raw RT-qPCR Ct values are also shown. Of note, the $E$ gene primer set did not bear LNA modifications and offers lower sensitivity, which could be useful for encoding log-scale quantitative information. n.d., not determined.

three false negatives. This latter effect, however, was not barcode dependent, as the second well for those samples (including identical LAMP-BCs but different PCR-BCs) was positive in each case. With this checkerboard experiment, we showed that (1) cross-contamination of adjacent wells can be expected to be minimal, and (2) LAMP-Seq generates sporadic dropouts.
Both potential issues can be addressed by requiring two of four replicates to be positive. To run four replicates on each sample (100 $\mu \mathrm{l}$ of RT-LAMP) using open-source components, the cost, excluding equipment depreciation, labor and licenses, amounts to US\$2.73 (as compared to US\$ 12.69 or the commercial protocol) (Supplementary Fig. 2). 

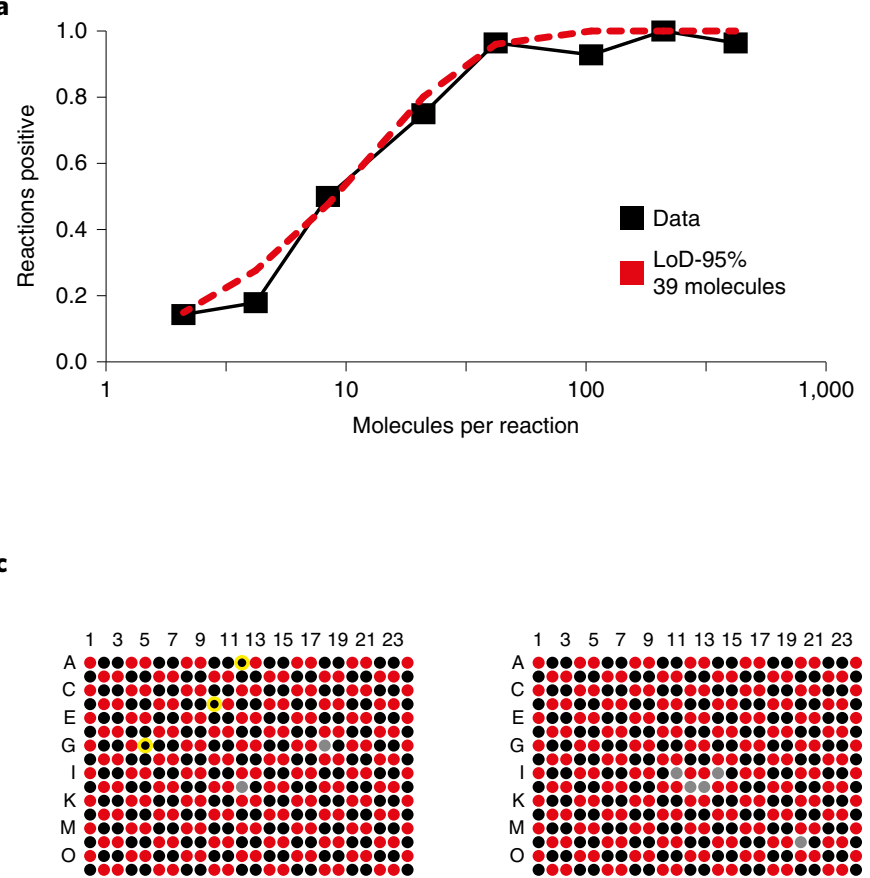

Barcode plate 1

$<1 \%$ of median positive reads

$1-10 \%$ of median positive reads

$>10 \%$ of median positive reads

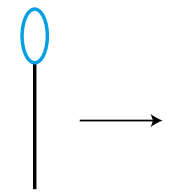

Oropharyngeal swab Barcode scanning (<1 min)

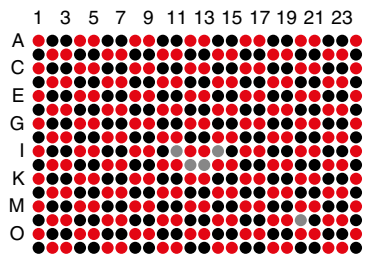

Barcode plate 2
LAMP barcode primers

RT-LAMP reaction

Water bath
Stabilization
Sterilization

$\left(95^{\circ} \mathrm{C}, 15 \mathrm{~min}\right)$

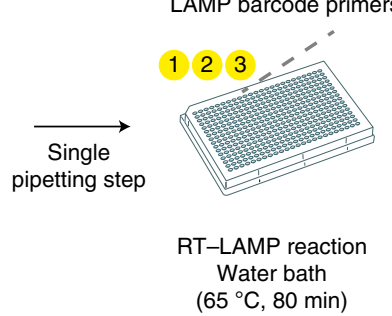

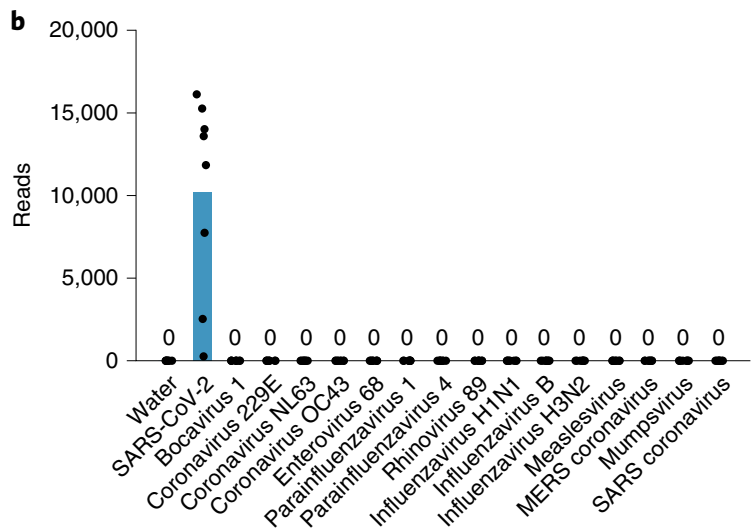

d

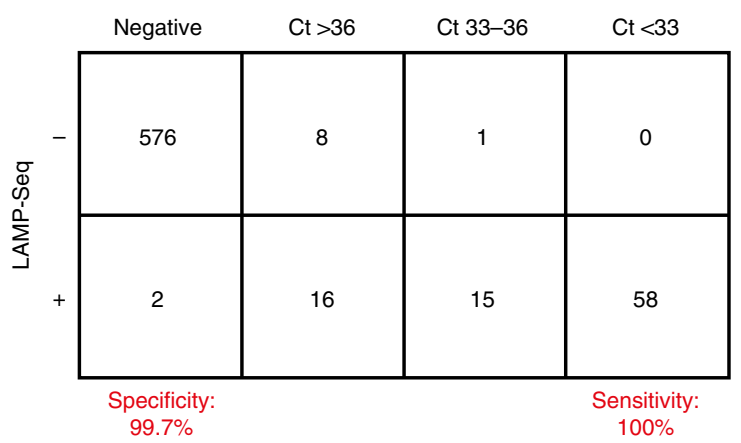

PCR barcode primers
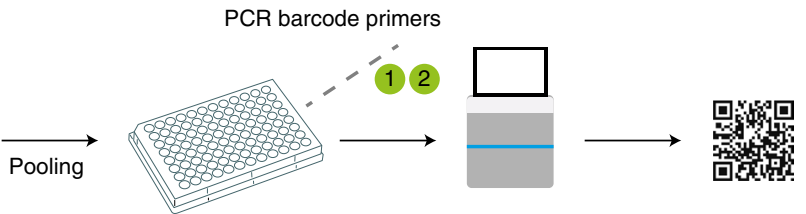

PCR

25 cycles

(80 min)
Deep sequencing
65 cycles

(5-10 h)

Data analysis Results to phone (1 h)

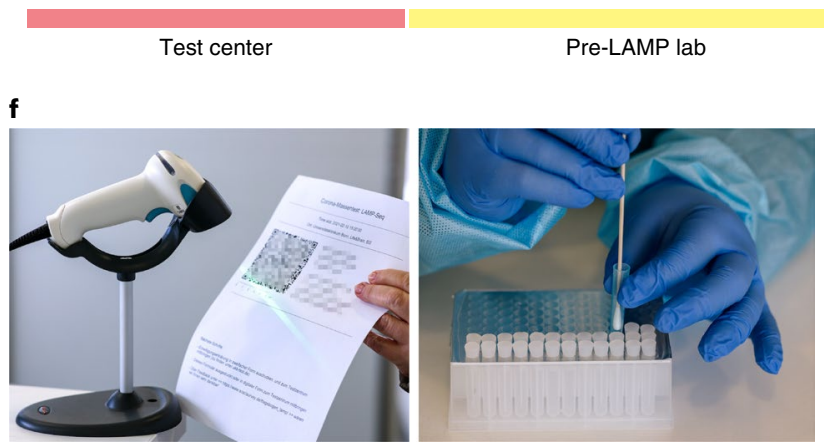

g
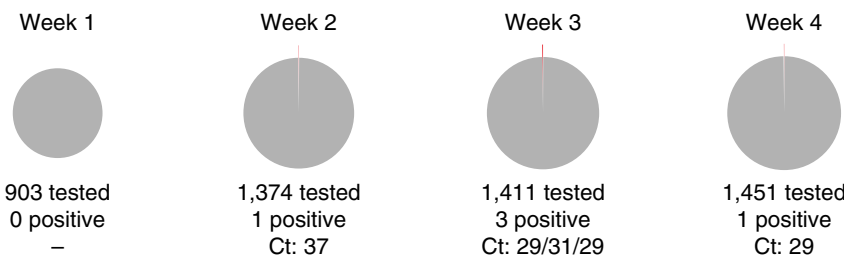

Fig. 3 | Validation of an open-source LAMP-Seq protocol. a, Estimation of the LoD-95 based on probit analysis of the fraction of positive replicates using a titration of chemically inactivated SARS-CoV-2 viral particles provided by the XPRIZE Foundation. $\mathbf{b}$, Specificity determination using IVT RNA of a panel of human virus genomes. c, $2 \times 384$ LAMP-Seq reactions alternatingly inoculated with IVT RNA (500,000 molecules per reaction) in a checkerboard pattern to evaluate barcoding specificity and cross-contamination. Yellow circles indicate false-negative replicates. $\mathbf{d}$, Summary of clinical validation study using open-source LAMP-Seq on 676 swab samples, performed on liquid-handling robots and analyzed in parallel by clinical RT-qPCR after RNA extraction from the same lysate. Samples annotated as $\mathrm{Ct}>36$ include late calls. e, Schematic outline of a proposed scalable testing procedure involving self-registration, semi-centralized barcoded RT-LAMP, pooling, sequencing and electronic results reporting. Colored bars indicate testing site (red), pre-LAMP (yellow) and post-LAMP (green) areas. f, Photographs showing the scanning process of individual QR codes generated during self-registration and 96-well lysis plates for immediate elution of virus particles from oropharyngeal swabs without cross-contamination of wells (plate is pre-sealed; swabs are inserted into wells using a disposable funnel; and then the well is covered with a silicone plug). g, Summary statistics from a 4-week-long pilot study of open-source LAMP-Seq for SARS-CoV-2 detection. In total, 5,139 asymptomatic volunteers were tested, of whom five were found to be positive in at least two replicates and subsequently confirmed by clinical RT-qPCR from the same lysate (Ct values shown below). The average time spent per person in our testing center was 2 min. 
We validated open-source LAMP-Seq on 676 residual swab samples from clinical testing, with four replicates per sample and using automated liquid handling. One positive (IVT RNA) and one negative control was included on each 96-well plate. LAMP-Seq displayed a sensitivity of $100 \%$ (with two or more positive replicates) among positive samples with corresponding Ct values $<33$ (Fig. 3d, right), in concordance with our LoD estimates. Among 578 samples that were negative by RT-qPCR, three were detected as positive by LAMP-Seq, one of which was subsequently confirmed to be a true positive using a more sensitive RT-qPCR protocol (Methods). Of 16 samples with $\mathrm{Ct}$ values between 33 and 36, 15 samples were identified as positive in LAMP-Seq, whereas 24 weakly positive samples (Ct above 36) were detected stochastically (Fig. 3d, middle).

Finally, we implemented LAMP-Seq in an end-to-end workflow (Fig. 3e) that was tested in a pilot study among medical and non-medical staff at the University Hospital Bonn (Application Note and Fig. 3f). Briefly, 96-well lysis plates were filled with open-source lysis buffer and controls, heat sealed with a pierceable foil and transported to the testing site. After self-registration, participants presented at the center. Supported by a visual and audio software, trained staff pierced one position on the plate with a disposable funnel device, took the oropharyngeal swab and then introduced it through the funnel into the well. After submerging for $10 \mathrm{~s}$, the swab was removed together with the funnel, and the well was then closed with a silicone plug. After transport to the pre-LAMP lab, lysates were sterilized in a thermoblock at $95^{\circ} \mathrm{C}$, and $8.3 \mu \mathrm{l}$ of lysed sample was stamped into a pre-made, barcoded RT-LAMP 384-well plate. This is the only mandatory liquid transfer step per sample. In the post-LAMP lab, plates were heated in a water bath to $65^{\circ} \mathrm{C}$ and subsequently pooled using either multi-channel pipetting or centrifugation (Methods). After small-scale PCRs on individual pools, the library was loaded on a MiSeq device. In total, LAMP-Seq was performed within $12 \mathrm{~h}$ ( $2 \mathrm{~h}$ swab to lab; $4 \mathrm{~h}$ inactivation and LAMP-based library prep; $5 \mathrm{~h}$ sequencing; and $0.5 \mathrm{~h}$ analysis). For samples with at least two of four positive replicates, the sample was located on the original 96-well lysis plate and entered into clinical qPCR testing. Positive test results were reported to the participant and health authorities. Negative results were reported to each tested individual digitally, based on an individual QR barcode (Application Note). Within 4 weeks, we tested 5,139 samples and identified five positive samples, all of which were confirmed by qPCR from remaining lysate (Ct values: 29-37; Fig. 3g).

\section{Discussion}

We have shown here the feasibility of deploying LAMP-Seq for population testing. By employing sample-specific barcodes at the first step of the protocol after sample lysis, large-scale pooling of samples can be achieved during downstream processing. Additionally, early pooling in combination with an isothermal amplification step reduces supply chain issues and the requirements for technical infrastructure. Our protocol also circumvents the requirement for RNA extraction. Using LNA modifications and further protocol optimization, we achieved an assay sensitivity and target specificity approaching that of the current gold standard qPCR, at considerably lower cost. LAMP-Seq uses existing NGS infrastructure to deliver results at scale, in theory ranging from several hundreds to thousands of samples per day per sequencing facility, and it could be deployed in developing countries. LAMP-Seq allows multiplexing of several target sequences in a single reaction, which enables scalable differential diagnosis of a multitude of pathogens (for example, influenza) or, alternatively, the detection of specific virus variants within one reaction. Future developments will include determination of its compatibility with other types of human samples (for example, saliva ${ }^{21}$ ) and combination with a fast read-out at point of care $^{22}$ to help identify the most scalable solution for unsupervised at-home sample collection. For such a decentralized deployment scenario, it will be favorable to have tens of thousands of unique barcodes. To minimize expected costs for primer synthesis and validation, we simulated a compressed barcode space where each LAMP reaction gets more than one barcode and also described the possibility for additional barcodes in the backward inner primer (BIP) (Supplementary Notes and Supplementary Fig. 3). Finally, the inclusion of UDG/UTP in the LAMP-Seq protocol could be envisaged to help reduce amplicon-driven contamination ${ }^{23,24}$ in situations where distinct pre-LAMP and post-LAMP areas are not available or sequencing of replicates is not possible. Once established, LAMP-Seq infrastructure could rapidly counter future waves of viral spread or novel pandemic outbreaks.

\section{Online content}

Any methods, additional references, Nature Research reporting summaries, source data, extended data, supplementary information, acknowledgements, peer review information; details of author contributions and competing interests; and statements of data and code availability are available at https://doi.org/10.1038/ s41587-021-00966-9.

Received: 20 October 2020; Accepted: 26 May 2021;

Published online: 29 June 2021

\section{References}

1. Dong, E., Du, H. \& Gardner, L. An interactive web-based dashboard to track COVID-19 in real time. Lancet. Infect. Dis. 20, 533-534 (2020).

2. Bai, Y. et al. Presumed asymptomatic carrier transmission of COVID-19. JAMA 323, 1406-1407 (2020).

3. Taipale, J., Romer, P. \& Linnarsson, S. Population-scale testing can suppress the spread of COVID-19. Preprint at medRxiv https://doi. org/10.1101/2020.04.27.20078329 (2020).

4. Peto, J. Covid-19 mass testing facilities could end the epidemic rapidly. Brit. Med. J. 368, m1163 (2020).

5. Mina, M. J. \& Andersen, K. G. COVID-19 testing: one size does not fit all. Science 371, 126-127 (2021).

6. Prince-Guerra, J. L. et al. Evaluation of Abbott BinaxNOW Rapid Antigen Test for SARS-CoV-2 infection at two community-based testing sites - Pima County, Arizona, November 3-17, 2020. MMWR. Morb. Mortal. Wkly Rep. 70, 100-105 (2021); erratum 70, 144 (2021).

7. Bloom, J. S. et al. Swab-Seq: a high-throughput platform for massively scaled up SARS-CoV-2 testing. Preprint at medRxiv https://doi.org/10.1101/ 2020.08.04.20167874 (2021)

8. Dao Thi, V. L. et al. A colorimetric RT-LAMP assay and LAMP-sequencing for detecting SARS-CoV-2 RNA in clinical samples. Sci. Transl. Med. 12, eabc7075 (2020)

9. James, P. et al. LamPORE: rapid, accurate and highly scalable molecular screening for SARS-CoV-2 infection, based on nanopore sequencing. Preprint at medRxiv https://doi.org/10.1101/2020.08.07.20161737 (2020).

10. Chappleboim, A. et al. ApharSeq: an extraction-free early-pooling protocol for massively multiplexed SARS-CoV-2 detection. Preprint at medRxiv https://doi.org/10.1101/2020.08.08.20170746 (2020).

11. Yelagandula, R. et al. Multiplexed detection of SARS-CoV-2 and other respiratory infections in high throughput by SARSeq. Nat. Commun. 12, 3132 (2021).

12. Nagamine, K., Hase, T. \& Notomi, T. Accelerated reaction by loop-mediated isothermal amplification using loop primers. Mol. Cell. Probes 16, 223-229 (2002)

13. Notomi, T. et al. Loop-mediated isothermal amplification of DNA. Nucleic Acids Res. 28, E63 (2000).

14. Mori, Y. \& Notomi, T. Loop-mediated isothermal amplification (LAMP): a rapid, accurate, and cost-effective diagnostic method for infectious diseases J. Infect. Chemother. 15, 62-69 (2009).

15. Broughton, J. P. et al. CRISPR-Cas12-based detection of SARS-CoV-2. Nat. Biotechnol. 38, 870-874 (2020).

16. Levenshtein, V. I. Binary codes capable of correcting deletions, insertions, and reversals. Sov. Phys. Dokl. 10, 707-710 (1966).

17. Braasch, D. A. \& Corey, D. R. Locked nucleic acid (LNA): fine-tuning the recognition of DNA and RNA. Chem. Biol. 8, 1-7 (2001).

18. Corman, V. M. et al. Detection of 2019 novel coronavirus (2019-nCoV) by real-time RT-PCR. Euro. Surveill. 25, 2000045 (2020).

19. Joung, J. et al. Detection of SARS-CoV-2 with SHERLOCK One-Pot Testing. N. Engl. J. Med. 383, 1492-1494 (2020). 
20. Kiefer, J. R. et al. Crystal structure of a thermostable Bacillus DNA polymerase I large fragment at $2.1 \AA ̊$ resolution. Structure 5, 95-108 (1997).

21. Lalli, M. A. et al. Rapid and extraction-free detection of SARS-CoV-2 from saliva by colorimetric reverse-transcription loop-mediated isothermal amplification. Clin. Chem. 67, 415-424 (2021).

22. Wu, Q. et al. INSIGHT: a population-scale COVID-19 testing strategy combining point-of-care diagnosis with centralized high-throughput sequencing. Sci. Adv. 7, eabe5054 (2021)

23. Fallahi, S. et al. An advanced uracil DNA glycosylase-supplemented loop-mediated isothermal amplification (UDG-LAMP) technique used in the sensitive and specific detection of Cryptosporidium parvum, Cryptosporidium hominis, and Cryptosporidium meleagridis in AIDS patients. Diagn. Microbiol. Infect. Dis. 91, 6-12 (2018).

24. Hsieh, K., Mage, P. L., Csordas, A. T., Eisenstein, M. \& Soh, H. T.

Simultaneous elimination of carryover contamination and detection of DNA with uracil-DNA-glycosylase-supplemented loop-mediated isothermal amplification (UDG-LAMP). Chem. Commun. 50, 3747-3749 (2014).

Publisher's note Springer Nature remains neutral with regard to jurisdictional claims in published maps and institutional affiliations.

(c) The Author(s), under exclusive licence to Springer Nature America, Inc. 2021, corrected publication 2022 


\section{Methods}

All clinical specimens either were collected upon informed consent under a human subjects protocol approved by the ethics committee of the Medical Faculty of the University Hospital Bonn (149/20 and 500/20) or were left-over specimens from viral diagnostic testing. No data on age, gender or ethnicity were collected.

\section{LAMP-Seq testing for SARS-CoV-2 using commercial buffers and enzymes.}

1. An inoculated cotton dry swab (nerbe plus, 09-819-5000) is inserted into $500 \mu \mathrm{l}$ of QuickExtract (Lucigen, QE09050) supplemented with $2 \mathrm{ng} \mathrm{\mu l}^{-1}$ of RNase-free plasmid DNA (pX330, Addgene, no. 42230) in a 15-ml Falcon tube or a $1.5-\mathrm{ml}$ microcentrifuge tube, incubated for at least $10 \mathrm{~min}$ at room temperature and heated to $95^{\circ} \mathrm{C}$ for $5 \mathrm{~min}$.

2. Lysate $(100 \mu \mathrm{l})$ is incubated with $35 \mathrm{mg}$ of activated carbon and ion-exchange beads for $30 \mathrm{~min}$, and $90 \mu \mathrm{l}$ of the mixture is transferred to a fresh tube. The $\mathrm{pH}$ of the lysis buffer is adjusted by adding $2.25 \mu \mathrm{l}$ of $1 \mathrm{~N} \mathrm{HCl}$.

3. A LAMP-Seq Master Mix for 110 reactions (including $10 \%$ overage) is prepared on ice, containing:

a. 1,147 $\mu$ l of LAMP Master Mix (New England Biolabs, E1700L)

b. $516 \mu \mathrm{l}$ of $1 \mathrm{M}$ Tris- $\mathrm{HCl} \mathrm{pH} 8.6$

c. $33.4 \mu \mathrm{l}$ of C-BIP primer (CGCATTGGCATGGAAGTCACTTTGATG GCACCTGTGTAG; $100 \mu \mathrm{M}$, HPLC-purified, IDT)

d. $4.2 \mu \mathrm{l}$ of C-F3-LNA primer $(\mathrm{A}+\mathrm{AC}+\mathrm{AC}+\mathrm{AA}+\mathrm{GC}+\mathrm{TTTCGGCAG}$ $100 \mu \mathrm{M}$, HPLC-purified, IDT; +denotes LNA modification in subsequent position)

e. $4.2 \mu \mathrm{l}$ of $\mathrm{C}-\mathrm{B} 3-\mathrm{LNA}$ primer $(\mathrm{G}+\mathrm{AA}+\mathrm{AT}+\mathrm{TT}+\mathrm{GG}+\mathrm{ATCTTTGT}-$ CATCC; $100 \mu \mathrm{M}$, HPLC-purified, IDT; + denotes LNA modification in subsequent position)

f. $8.3 \mu \mathrm{l}$ of C-LF primer (TTCCTTGTCTGATTAGTTC; $100 \mu \mathrm{M}$, HPLCpurified, IDT)

g. $8.3 \mu \mathrm{l}$ of C-LB primer (ACCTTCGGGAACGTGGTT; $100 \mu \mathrm{M}$, HPLCpurified, IDT)

h. $6.7 \mu \mathrm{l}$ of ACTB-BIP primer (CTGAACCCCAAGGCCAACCGGCTG GGGTGTTGAAGGTC; $100 \mu \mathrm{M}$, IDT)

i. $0.83 \mu \mathrm{l}$ of ACTB-F3 primer (AGTACCCCATCGAGCACG; $100 \mu \mathrm{M}$, IDT)

j. $0.83 \mu \mathrm{l}$ of ACTB-B3 primer (AGCCTGGATAGCAACGTACA; $100 \mu \mathrm{M}$, IDT)

k. $1.67 \mu \mathrm{l}$ of ACTB-LF primer (TGTGGTGCCAGATTTTCTCCA; $100 \mu \mathrm{M}$, IDT)

1. $1.67 \mu$ of ACTB-LB primer (CGAGAAGATGACCCAGATCATGT; $100 \mu \mathrm{M}, \mathrm{IDT})$

m.2.3 $\mu \mathrm{l}$ of RNase-free plasmid DNA (pX330, $1 \mu \mathrm{g} \mu \mathrm{l}^{-1}$, Addgene, no 42230)

n. $57.3 \mu \mathrm{l}$ of Bst 3.0 (8,000 units per ml, New England Biolabs, M0374L)

4. 25- $\mu \mathrm{l}$ LAMP-Seq reactions containing the following components are assembled in a 384-well plate:

a. $16.2 \mu \mathrm{l}$ of LAMP-Seq Master Mix

b. $0.5 \mu \mathrm{l}$ of an aqueous solution of:

i. barcoded C-FIP (TGCGGCCAATGTTTGTAATCAG

NNNNNNNNNNCCAAGGAAATTTTGGGGAC, where Ns denote a barcode sequence; $60 \mu \mathrm{M}$, IDT)

ii. barcoded ACTB-FIP (GAGCCACACGCAGCTCATTGTA

NNNNNNNNNNTCACCAACTGGGACGACA, where Ns denote a barcode sequence; $12 \mu \mathrm{M}$, IDT)

c. $8.3 \mu \mathrm{l}$ of swab lysate from Step 2

5. The RT-LAMP plate is thoroughly sealed using foil (BIOplastics, no. 157300), and the plate is submerged in a $65^{\circ} \mathrm{C}$ water bath for $80 \mathrm{~min}$, avoiding air bubbles under the plate.

6. Reactions are pooled on ice. If replicates of one sample have identical barcodes, a multi-channel pipette has to be used ( 0.25 pipette tips per sample). If each replicate on the 384-well LAMP plate has individual barcodes, pooling can be performed by brief top-down centrifugation into a disposable container (for example, pipette box lid).

7. The pool is diluted 1:100,000 in double distilled water (together with the subsequent dilution of 1 in 10 at the PCR stage; this results in a final dilution of the pool of 1 in 1,000,000).

8. For each pool, an 18-cycle 50- $\mu$ PCR reaction is performed:

a. $25 \mu$ l of NEBNext $2 \times$ Master Mix (New England Biolabs)

b. $0.25 \mu \mathrm{l}$ of PCR-C-fwd primer (ACACTCTTTCCCTACACGACGCTC TTCCGATCTAACGCTGAAGCGCTGGGGGCAAA; $100 \mu \mathrm{M}$, IDT)

c. $0.25 \mu \mathrm{l}$ of PCR-C-rev primer (TGACTGGAGTTCAGACGTGTGCTC TTCCGATCTGTTTGTAATCAGTTCCTTGTCTG; $100 \mu \mathrm{M}$, IDT)

d. $5 \mu$ l of diluted RT-LAMP reaction

e. $19.5 \mu \mathrm{l}$ of water

f. PCR cycle conditions: $20 \mathrm{~s}$ at $98^{\circ} \mathrm{C}, 20 \mathrm{~s}$ at $65^{\circ} \mathrm{C}$ and $30 \mathrm{~s}$ at $72{ }^{\circ} \mathrm{C}$

9. For each pool, a secondary 18 -cycle $50-\mu \mathrm{l}$ PCR reaction is performed with: a. $25 \mu \mathrm{l}$ of NEBNext $2 \times$ Master Mix (New England Biolabs)

b. $0.25 \mu \mathrm{l}$ of pool-specific fwd barcoding primer (AATGATACGGCGAC CACCGAGATCTACACNNNNNNNNNNACACTCTTTCCCTACAC GACGCT, where Ns denote a specific barcode sequence; $100 \mu \mathrm{M}$, IDT)

c. $0.25 \mu \mathrm{l}$ of pool-specific rev barcoding primer (CAAGCAGAAGACGG CATACGAGATNNNNNNNNNNGTGACTGGAGTTCAGACGTGT GCT, where Ns denote a specific barcode sequence; $100 \mu \mathrm{M}$, IDT)

d. $5 \mu$ l of the previous PCR reaction

e. $19.5 \mu$ l of water

f. PCR cycle conditions: $20 \mathrm{~s}$ at $98^{\circ} \mathrm{C}, 20 \mathrm{~s}$ at $65^{\circ} \mathrm{C}$ and $30 \mathrm{~s}$ at $72{ }^{\circ} \mathrm{C}$ of note, Steps 8 and 9 can also be combined into a single PCR reaction as described for the open-source protocol (Step 8).

10. The PCR products are pooled on ice, cut from a $2 \%$ agarose E-Gel, purified twice using a silica spin column (Qiagen), quantified using a NanoDrop photospectrometer (Thermo Fisher Scientific) and sequenced on an Illumina MiSeq or iSeq sequencer.

11. Using the LAMP-Seq Inspector software (http://manuscript.lamp-seq. org/Inspector.htm), barcodes co-occurring with the correct viral genome sequence, excluding sequence portions covered by primers, are determined. This analysis can also be performed using a 'kallisto | bustools' workflow ${ }^{25}$.

\section{LAMP-Seq testing for SARS-CoV-2 using open-source buffers and enzymes.}

1. The following lysis buffer LSB is prepared and stored on ice:
a. $300 \mathrm{mM}$ Tris- $\mathrm{HCl} \mathrm{pH} 8.5$
b. 2.7 units per $\mathrm{ml}$ of proteinase $\mathrm{K}$ (NEB, P8107S)
c. $2.5 \mathrm{M}$ betaine (Sigma-Aldrich, $61962-50 \mathrm{G}$ )

2. Dry swabs from routine clinical testing are inserted into a 96-well 1-ml deep-well plate (Eppendorf, 0030501217) containing $700 \mu \mathrm{l}$ of LSB lysis buffer and, optionally, $175 \mu \mathrm{l}$ of a dry volume of activated carbon and weak acid cation exchange beads, incubated for at least $10 \mathrm{~s}$ at room temperature and heated to $95^{\circ} \mathrm{C}$ for up to $15 \mathrm{~min}$.

3. A LAMP-Seq Master Mix for six 384-well plates (including overage) is prepared on ice, containing:

a. $8 \mathrm{ml}$ of isothermal reaction buffer (New England Biolabs, B0537S)

b. $2.8 \mathrm{ml}$ of dNTP mix $10 \mathrm{mM}$ (NEB, N0447L)

c. $17.92 \mathrm{ml}$ of $1 \mathrm{M}$ Tris- $\mathrm{HCl} \mathrm{pH} 8.5$

d. $4.8 \mathrm{ml}$ of $\mathrm{MgSO}_{4} 100 \mathrm{mM}$ (NEB, B1003S)

e. $320 \mu \mathrm{l}$ of C-BIP primer (CGCATTGGCATGGAAGTCACTTTGAT GGCACCTGTGTAG; $100 \mu \mathrm{M}$, IDT)

f. $160 \mu \mathrm{l}$ of C-F3-LNA primer $(\mathrm{A}+\mathrm{AC}+\mathrm{AC}+\mathrm{AA}+\mathrm{GC}+\mathrm{TTTCGGCAG}$; $100 \mu \mathrm{M}, \mathrm{IDT} ;+$ stands for LNA modification in subsequent position)

g. $160 \mu \mathrm{l}$ of C-B3-LNA primer $(\mathrm{G}+\mathrm{AA}+\mathrm{AT}+\mathrm{TT}+\mathrm{GG}+\mathrm{ATCTTTGT}-$ CATCC; $100 \mu \mathrm{M}$, IDT; +stands for LNA modification in subsequent position)

h. $320 \mu \mathrm{l}$ of C-LF primer (TTCCTTGTCTGATTAGTTC; $100 \mu \mathrm{M}$, IDT)

i. $320 \mu \mathrm{l}$ of C-LB primer (ACCTTCGGGAACGTGGTT; $100 \mu \mathrm{M}$, IDT)

j. $80 \mu \mathrm{l}$ of pUC19 plasmid DNA $\left(1 \mu \mathrm{g} \mathrm{ul}^{-1}\right.$, New England Biolabs N3041L)

k. $12.16 \mathrm{ml}$ of water

1. $3.2 \mathrm{ml}$ of wild-type Bst-LF-Idaho polymerase $\left(1 \mathrm{mg} \mathrm{ml}^{-1}\right)$.

4. 25- $\mu$ LAMP-Seq reactions containing the following components are assembled in a 384-well plate:

a. $15.7 \mu$ l of LAMP-Seq Master Mix

b. $1 \mu \mathrm{l}$ of barcoded C-FIP (TGCGGCCAATGTTTGTAATCAG-NN NNNNNNNN-CCAAGGAAATTTTGGGGAC, where Ns denote a barcode sequence; $10 \mu \mathrm{M}$, IDT)

c. $8.3 \mu \mathrm{l}$ of swab lysate from Step 2 .

Of note, we observed a similar sensitivity when scaling down to $12.5 \mu \mathrm{l}$ per reaction in a 384-well plate.

1. Plates are submerged in a water bath at $65^{\circ} \mathrm{C}$ for $80 \mathrm{~min}$, protected by two nested Ziploc bags.

2. Reactions are pooled on ice. If replicates of one sample have identical barcodes, a multi-channel pipette has to be used ( 0.25 pipette tips per sample). If each replicate on the 384-well LAMP plate has individual barcodes, pooling can be performed by brief top-down centrifugation into a disposable container (for example, pipette box lid).

3. The pool is diluted 1:40,000 in double distilled water (together with the subsequent dilution of 1 in 10 at the PCR stage; this results in a final dilution of the pool of 1 in 400,000).

4. One-step PCR reactions are performed $\left(25\right.$ cycles, $\left.\mathrm{Ta}=65^{\circ} \mathrm{C}\right)$ containing:

a. $12.5 \mu \mathrm{l}$ of NEBNext $2 \times$ Master Mix (New England Biolabs)

b. $2.5 \mu \mathrm{l}$ of diluted LAMP pool

c. $2.5 \mu \mathrm{l}$ of primer mix, containing:

i. $5 \mu \mathrm{M}$ unique fwd primer (AATGATACGGCGACCACCGA GATCTACAC-NNNNNNNNNN-ACACTCTTTCCCTACAC 
GACGCTCTTCCGATCTAACGCTGAAGCGCTGGGGGCAAA,

where Ns denote a barcode sequence; Ultramer, IDT)

ii. $5 \mu \mathrm{M}$ unique rev primer (CAAGCAGAAGACGGCATACGAG AT-NNNNNNNNNN-GTGACTGGAGTTCAGACGTGTGCTC TTCCGATCTGTTTGTAATCAGTTCCTTGTCTG, where Ns denote a barcode sequence; Ultramer, IDT)

$$
\text { a. } 7.5 \mu \text { of water }
$$

9. $\quad$ PCR products are pooled and purified using a Qiagen PCR spin purification column and eluted in $30 \mu \mathrm{l}$ of water.

10. The library band at $258 \mathrm{bp}$ is gauged against a $3 \times$ dilution series of a previously run reference library using a $2 \%$ Agarose EX E-Gel (Thermo Fisher Scientific).

11. A MiSeq Nano kit (Illumina, MS-103-1001) is loaded according to the manufacturer's protocol, with 10\% PhiX spike-in (Illumina) and the following cycle numbers:
a. 100 cycles read- 1
b. 8 cycles index-1
c. 8 cycles index- 2

\section{Sequencing on the iSeq platform.}

1. An N-100 random-index library is generated by NEBNext PCR (NEB) using template oligo iSeq-N100 (Supplementary Data 1; Ultramer, IDT), and primers iSeq-N100-fwd and iSeq-N100-rev (Supplementary Data 1; IDT). PCR products are purified using a silica-based PCR purification kit (Qiagen) and quantified using a NanoDrop photospectrometer (Thermo Fisher Scientific).

2. The library band at $258 \mathrm{bp}$ is gauged against a $3 \times$ dilution series of a previously run reference library using a $2 \%$ Agarose EX E-Gel (Thermo Fisher Scientific).

3. An iSeq v2 reagent cartridge (20031371, Illumina) is thawed in a water bath a room temperature for $1 \mathrm{~h}$; the flow cell is inserted; and the kit is kept at room temperature. The LAMP-Seq library and the N-100 random-index library are diluted to $2 \mathrm{nM}$ each in water. Next, $1 \mu \mathrm{l}$ of the combined library is mixed with $150 \mu \mathrm{l}$ of Tris $\mathrm{pH} 8.5$. Then, $20 \mu \mathrm{l}$ of the final dilution is loaded into the reagent cartridge and sequenced according to the manufacturer's instruction using the following cycle numbers:
a. 100 cycles read-1
a. 8 cycles index-1
a. 8 cycles index-2

\section{Checkerboard validation of 96 LAMP-Seq barcodes.}

1. A 384-well LAMP-Seq plate is prepared using a Beckman Coulter FXP pipetting robot, following the open-source LAMP-Seq protocol. Wells contain all reaction components except template (total: $16.7 \mu \mathrm{l}$ per well), and 96 barcoded primers are tested per plate, as each primer is spotted to four adjacent wells.

2. A dilution of IVT-generated template RNA with 60,200 molecules per $\mu \mathrm{l}$ in water is created.

3. In the first row of a 96-well PCR plate, $300 \mu \mathrm{l}$ of template RNA dilution is spotted, alternating with water.

4. Using a 12-channel pipette, $8.3 \mu \mathrm{l}$ of template or water (amounting to 500,000 or 0 molecules per LAMP reaction) are spotted to quadrants 1 and 4 of the LAMP-Seq plate and mixed four times without performing a blow-out.

5. The template plate is turned $180^{\circ}$, and $8.3 \mu \mathrm{l}$ of template or water is spotted to quadrants 2 and 3 of the LAMP-Seq plate and mixed four times without performing a blow-out.

6. The LAMP-Seq plate is heated to $65^{\circ} \mathrm{C}$ for $80 \mathrm{~min}$ and pooled, diluted, amplified and sequenced on a MiSeq platform (Illumina) as described in the above open-source protocol.

Clinical RT-qPCR pipeline. Swabs were rehydrated in $600 \mu \mathrm{ml}^{-1}$ of PBS, saline or LAMP-Seq lysis buffer. Viral RNA was extracted using the chemagic Prime viral DNA/RNA 300 kit (PerkinElmer) on a chemagic Prime 8 system (PerkinElmer). The viral sample $(150-290 \mu \mathrm{l})$ was mixed with $10 \mu \mathrm{l}$ of the internal control sample and $300 \mu \mathrm{l}$ of lysis buffer. Extraction was performed according to the manufacturer's protocol, and viral RNA was eluted in $45 \mu \mathrm{l}$ of elution buffer for subsequent analysis. Detection of viral RNA using one-step real-time RT-PCR was performed according to Corman et al. ${ }^{18}$ with the iTaq Universal Probes One-Step Kit (Bio-Rad), using $5 \mu \mathrm{l}$ of eluate per reaction and primers and probes against the E gene (E_Sarbeco_F1: ACAGGTACGTTAATAGTTAATAGCGT, E_Sarbeco_R2: ATATTGCAGCAGTACGCACACA and E_Sarbeco_P1: FAM-ACACTAGCCAT CCTTACTGCGCTTCG-BBQ; TIB MolBiol). Spike-in RNA of the bacteriophage MS2 served as an internal control and was detected using the Luna Universal Probe One-Step RT-qPCR Kit (New England Biolabs) using $2 \mu$ lof eluate and corresponding primers and probes (MS2_F: TGCTCGCGGATACCCG, MS2_R: AACTTGCGTTCTCGAGCGAT and MS2_P: YAK-ACCTCGGGTTTCC
GTCTTGCTCGT-BBQ; TIB MolBiol). The reactions for the $E$ gene and internal control were performed using dual detection of FAM and YAK/VIC in a LightCycler 480 (Roche).

Clinical RT-qPCR protocol 2 (high sensitivity). Next, $20 \mu$ of extracted viral RNA (see clinical RT-qPCR pipeline) was analyzed using the New Coronavirus Nucleic Acid Detection Kit (PerkinElmer), according to the manufacturer's protocol, in a total sample volume of $30 \mu \mathrm{l}$. Samples were analyzed using QuantStudio (Thermo Fisher Scientific; comparative Ct method of three fluorophores: internal control: VIC; $N$ gene: FAM; and ORF1b: ROX).

Viruses and cells. The SARS-CoV-2 strain MUC-IMB-1 was isolated and kindly supplied by Rosina Ehmann and Gerhard Dobler (Bundeswehr Institute of Microbiology). The virus was propagated and titrated on Vera E6 cells (ATCC CRL-1586). All work with SARS-CoV-2 was conducted in a Biosafety Level (BSL)-3 facility in accordance with the biosafety guidelines of the Israel Institute for Biological Research (IIBR). VSV serotype Indiana, kindly provided by Eran Bacharach (Tel-Aviv University), was propagated and titrated on Vero cells (ATCC CCL-81). All work with VSV was conducted in a BSL-2 facility in accordance with the biosafety guidelines of the IIBR

Lysis buffer inactivation assay. QuickExtract DNA extraction solution (Lucigen) was tested in accordance with the manufacturer's suggested buffer-to-sample ratio. Universal transfer medium (UTM; Copan) aliquots were inoculated with either $5 \times 10^{6}$ plaque-forming units (PFU) per ml of SARS-CoV- 2 or $2 \times 10^{9} \mathrm{PFU}$ per $\mathrm{ml}$ of VSV and incubated at $22^{\circ} \mathrm{C}, 65^{\circ} \mathrm{C}$ or $95^{\circ} \mathrm{C}$ for $10-30 \mathrm{~min}$. Positive and negative control samples included UTM inoculated with viable virus without lysis buffer and UTM inoculated with lysis buffer without virus, respectively. The LoD was defined as the first serial dilution of the negative control that did not cause a cytopathic effect (CPE) by itself (represented in log scale). Briefly, Vero E6 cells (for SARS-CoV-2) or Vero cells (for VSV) were cultured in DMEM supplemented with $10 \%$ FBS, MEM non-essential amino acids, $2 \mathrm{mM} \mathrm{L}$-glutamine, $100 \mathrm{U} \mathrm{ml}^{-1}$ of penicillin, $0.1 \mathrm{mg} \mathrm{ml}^{-1}$ streptomycin and $12.5 \mathrm{U} \mathrm{ml}^{-1}$ of nystatin (Biological Industries). Monolayers $\left(2.5 \times 10^{5}\right.$ cells per well in 24 -well plates $)$ were washed once with MEM Eagle medium without FBS and infected with $200 \mu \mathrm{l}$ of ten-fold serial dilutions of the samples. After $1 \mathrm{~h}$ of incubation, the wells were overlaid with $1 \mathrm{ml}$ of MEM medium containing 2\% FCS, MEM non-essential amino acids, $2 \mathrm{mM}$ L-glutamine, $100 \mathrm{U} \mathrm{ml}^{-1}$ of penicillin, $0.1 \mathrm{mg} \mathrm{ml}^{-1}$ of streptomycin, $12.5 \mathrm{U} \mathrm{ml}^{-1}$ of nystatin and $0.15 \%$ sodium bicarbonate (Biological Industries). The cells were then incubated at $37^{\circ} \mathrm{C} / 5 \% \mathrm{CO}_{2}$ for $5 \mathrm{~d}$ (SARS-CoV-2) or $1 \mathrm{~d}$ (VSV). CPE was determined by counter-staining with crystal violet solution.

Bst-LF-Idaho polymerase protein expression and purification. Bst polymerase large fragment from a Geobacillus strain sampled in Idaho ${ }^{20}$ was cloned into a pET vector with an N-terminal His6-3C-tag (the full plasmid sequence is provided in Supplementary Data 1). Recombinant protein was expressed in Escherichia coli BL21 Rosetta (DE3) cells in TB autoinduction media supplemented with $17 \mathrm{mM}$ $\mathrm{KH}_{2} \mathrm{PO}_{4}, 72 \mathrm{mM} \mathrm{K}_{2} \mathrm{HPO}_{4}, 1.5 \%$ lactose, $0.05 \%$ glucose and $2 \mathrm{mM} \mathrm{MgSO}_{4}$ at $18^{\circ} \mathrm{C}$ overnight. Cells were harvested by centrifugation and resuspended in lysis buffer (50 mM Tris/ $\mathrm{HCl} \mathrm{pH} 8.0,1 \mathrm{M} \mathrm{NaCl}, 20 \mathrm{mM}$ imidazol and $10 \%$ glycerol) followed by sonication. The lysate was cleared in a Beckman Coulter Avanti JNX-26 centrifuge with a JA-25.50 rotor $\left(20,000\right.$ r.p.m. for $30 \mathrm{~min}$ at $\left.4{ }^{\circ} \mathrm{C}\right)$ and applied to a HisTrap FF column (GE Healthcare). After washing with 10 column volumes of lysis buffer, protein was eluted in elution buffer $(50 \mathrm{mM}$ Tris/ $\mathrm{HCl} \mathrm{pH} \mathrm{8.0,0.5} \mathrm{M}$ $\mathrm{NaCl}, 200 \mathrm{mM}$ imidazol and $10 \%$ glycerol). Fractions of the main peak were pooled and diluted 1:10 with IEX loading buffer $(20 \mathrm{mM}$ Tris/ $\mathrm{HCl}, 100 \mathrm{mM} \mathrm{NaCl}$ and $10 \%$ glycerol), and the affinity tag was removed using 1:100 3C protease overnight at $4{ }^{\circ} \mathrm{C}$. Protein was loaded onto a reverse HisTrap FF column coupled to a HiTrapQ HP column (GE Healthcare). After loading, the HisTrap column was removed, and protein was eluted from the HiTrapQ column with 25\% IEX elution buffer $(20 \mathrm{mM}$ Tris/ $\mathrm{HCl}, 1 \mathrm{M} \mathrm{NaCl}$ and $10 \%$ glycerol). Fractions of the main peak were pooled and diluted 1:5 with heparin loading buffer $(20 \mathrm{mM}$ Tris/ $\mathrm{HCl}, 100 \mathrm{mM} \mathrm{NaCl}$ and $10 \%$ glycerol). Sample was loaded onto a HiPrep heparin FF column (GE Healthcare) and eluted using $40 \%$ heparin elution buffer $(20 \mathrm{mM}$ Tris/ $\mathrm{HCl}, 1 \mathrm{M} \mathrm{NaCl}$ and $10 \%$ glycerol). Fractions of the main peak were concentrated using Amicon filters (Millipore) and applied to size exclusion chromatography using a Superdex 200 Prep Grade column (GE Healthcare) equilibrated with SEC buffer ( $25 \mathrm{mM}$ Tris/HCl pH 8.0 and $250 \mathrm{mM} \mathrm{KCl}$ ). Fractions of the main peak were pooled, concentrated to $1 \mathrm{mg} \mathrm{ml}^{-1}$ using Amicon filters and stored in 1-ml aliquots in storage buffer $(10 \mathrm{mM}$ Tris/ $\mathrm{HCl}$ pH 7.5, $100 \mathrm{mM} \mathrm{KCl,} 1 \mathrm{mM}$ DTT, $0.1 \mathrm{mM}$ EDTA and $50 \%$ glycerol) at $-20^{\circ} \mathrm{C}$.

Reporting Summary. Further information on research design is available in the Nature Research Reporting Summary linked to this article.

\section{Data availability}

Genomic sequences of SARS-CoV-2 are available at the National Center for Biotechnology Information (https://www.ncbi.nlm. nih.gov/sars-cov-2/). Read statistics and read counts are provided in Supplementary Data 2. Example 
LAMP-Seq data are available at the Sequence Read Archive (accession number PRJNA729981).

\section{Plasmid availability}

The expression plasmid pET-Bst-LF-Idaho is available from http://www.addgene. org/ (no. 170469).

\section{Code availability}

The LAMP-Seq Inspector tool for processing raw LAMP-Seq data is available at http://manuscript.lamp-seq. org/Inspector.htm. Python scripts (v3.6.0) for designing the error-correcting barcodes are available at https://github.com/ feldman4/dna-barcodes. Jupyter Notebooks for numerical simulations and MATLAB scripts (R2020a Update 4) for figure generation are available at https:// github.com/ dbli2000/SARS-CoV2- Bloom-Filter.

\section{References}

25. Booeshaghi, A. S. et al. Reliable and accurate diagnostics from highly multiplexed sequencing assays. Sci. Rep. 10, 21759 (2020).

\section{Acknowledgements}

Foremost, we thank all participating individuals who enabled this research by donating swab samples. We thank M. Knop, G. Smith, G. Hartmann, F. Heyder, P. Buckhaults, L. Pachter, Y. Dor, S. Strobel, S. Laber, A. Guo, A. Heimbach, A.-M. Eis-Hübinger, E. Zhang, V. Huang, D. Liu, A. Vijayakumar, S. Virreira Winter, J. Schmid-Burgk, S. Frank and J. M. Zapata Rolón for helpful discussions. We thank A. Englisch and P. Tessmann for help performing RNA extraction and RT-qPCR; C. Balzun, P. L. Scheid and R. M. Hagen for support during method development; and D. Hinze for plasmid preparation. We thank K. Remans and J. Flock for cloning of the expression plasmid for Bst-LF-Idaho polymerase. The template for Bst-LF-Idaho was a gift from A. D. Ellington. We thank A. Miller, S. Schwarz, S. Knösel and M. Schultz for support during the LAMP-Seq pilot study and S. Scheithauer, G. Faetkenheuer, U. Protzer and H. Streeck for grant coordination within NUM/B-FAST. Funding: M.G., M.H., M.M.N. and J.S.B. were supported by the Deutsche Forschungsgemeinschaft (DFG) under Germany's Excellence Strategy - EXC2151 - 390873048. K.U.L. is supported by the Emmy-Noether program of the DFG (LU1944/3-1), and the DFG also provided support to M.G. (GE 976/9-2). J.D.B. is supported by a Howard Hughes Medical Institute Gilliam Fellowship. The LAMP-Seq pilot study was supported by the B-FAST framework (Bundesweites Forschungsnetz Angewandte Surveillance und Testung) of the joint project Nationales Forschungsnetzwerk der Universitätsmedizin zu Covid-19, funded by the Federal Ministry for Education and Research under project number 01KX2021 (to J.S.B). K.U.L., M.M.N. and J.S.B. are members of the German COVID-19 OMICS initiative (https://decoi.eu/). A.R. and F.Z. are Investigators of the Howard Hughes Medical Institute. Work was supported by the Klarman Incubator (A.R.). F.Z. is supported by National Institutes of Health grants (1R01-HG009761, 1R01-MH110049 and 1DP1-HL141201); the Howard Hughes
Medical Institute; the Harold G. and Leila Mathers Foundation; the Patrick J. McGovern Foundation; the Edward Mallinckrodt, Jr. Foundation; the Open Philanthropy Project; the Poitras Center for Psychiatric Disorders Research at MIT; the Hock E. Tan and K. Lisa Yang Center for Autism Research at MIT; the Phillips family; and J. and P. Poitras.

\section{Author contributions}

K.U.L.: investigation, methodology, project administration and writing-original draft. R.M.S.: investigation, resources and project administration. D.L.: investigation and formal analysis. M. Jacobs: investigation. R.H.: investigation and methodology. K.B.: resources, software and project administration. J.L.: investigation. M. Słabicki: investigation. A.B.-S.: investigation. O.I.: investigation. S.W.: investigation. T.E.: investigation. N.P.: investigation. W.R.: investigation. G.W.: resources. D.F.: investigation. B.L.: methodology. N.I.: methodology. L.M.H.: methodology. E.B.: methodology. I.H.K.: resources. M. Schmitz: resources. A.W.: investigation. M.D.: resources. E.S.: resources. M. Jentzsch: project administration. J.D.B.: investigation. J.S.: investigation. J.R.: investigation. B.C.: investigation. M.G.: methodology and resources. M.H.: supervision. R.M.: supervision and writing. M.M.N.: supervision and funding acquisition. P.H.: investigation and methodology. M.E.: supervision. A.R.: supervision and funding acquisition. F.Z.: supervision and funding acquisition. J.L.S.-B.: conceptualization, project administration, software, supervision, funding acquisition, investigation and writing-original draft.

\section{Competing interests}

K.U.L., D.L., F.Z. and J.S.-B. are inventors on patent applications filed by the Broad Institute and others related to this work with the specific aim of ensuring that this technology can be made rapidly available for research and deployment. F.Z. is a co-founder of Editas Medicine, Beam Therapeutics, Pairwise Plants, Arbor Biotechnologies and Sherlock Biosciences. A.R. is a founder and equity holder of Celsius Therapeutics, an equity holder in Immunitas Therapeutics and, until 31 August 2020, was a scientific advisory board member of Syros Pharmaceuticals, Neogene Therapeutics, Asimov and Thermo Fisher Scientific. Since 1 August 2020, A.R. has been an employee of Genentech, a member of the Roche Group. P.H. and M.M.N. are scientific advisory board members of HMG Systems Bioengineering and receive salaries from Life \& Brain. M.M.N. served on scientific advisory boards for the Lundbeck Foundation and Robert-Bosch-Stiftung, was reimbursed for travel expenses by Shire and holds shares in Life \& Brain. J.D.B. is a co-founder of Coral Genomics and a scientific advisory board member of Alix Ventures.

\section{Additional information}

Supplementary information The online version contains supplementary material available at https://doi.org/10.1038/s41587-021-00966-9.

Correspondence and requests for materials should be addressed to J.L.S.-B.

Peer review information Nature Biotechnology thanks Charles Chiu and the other, anonymous, reviewer(s) for their contribution to the peer review of this work.

Reprints and permissions information is available at www.nature.com/reprints. 


\section{Reporting Summary}

Nature Research wishes to improve the reproducibility of the work that we publish. This form provides structure for consistency and transparency in reporting. For further information on Nature Research policies, see our Editorial Policies and the Editorial Policy Checklist.

\section{Statistics}

For all statistical analyses, confirm that the following items are present in the figure legend, table legend, main text, or Methods section.

$\mathrm{n} / \mathrm{a}$ Confirmed

$\bigotimes$ The exact sample size $(n)$ for each experimental group/condition, given as a discrete number and unit of measurement

$\square$ A statement on whether measurements were taken from distinct samples or whether the same sample was measured repeatedly

X The statistical test(s) used AND whether they are one- or two-sided

Xnly common tests should be described solely by name; describe more complex techniques in the Methods section.

$\triangle \square$ A description of all covariates tested

Х $\square$ A description of any assumptions or corrections, such as tests of normality and adjustment for multiple comparisons

A full description of the statistical parameters including central tendency (e.g. means) or other basic estimates (e.g. regression coefficient)

$\triangle \square$ AND variation (e.g. standard deviation) or associated estimates of uncertainty (e.g. confidence intervals)

$X$ For null hypothesis testing, the test statistic (e.g. $F, t, r$ ) with confidence intervals, effect sizes, degrees of freedom and $P$ value noted

Х Give $P$ values as exact values whenever suitable.

Х $\square$ For Bayesian analysis, information on the choice of priors and Markov chain Monte Carlo settings

Х $\square$ For hierarchical and complex designs, identification of the appropriate level for tests and full reporting of outcomes

Х $\square$ Estimates of effect sizes (e.g. Cohen's $d$, Pearson's $r$ ), indicating how they were calculated

\section{Our web collection on statistics for biologists contains articles on many of the points above.}

\section{Software and code}

Policy information about availability of computer code

Data collection Self-registration of probands was enabled by a pilot software that was GDPR-compliant. This software cannot be made available as it might qualify as a medical device, which falls under regulation procedures in some European countries.

Data analysis The LAMP-Seq Inspector tool for processing raw LAMP-Seq data is available at: http://manuscript.lamp-seq.org/Inspector.htm. Python scripts (v3.6.0) for designing the error-correcting barcodes are available at: https://github.com/feldman4/dna-barcodes. Jupyter Notebooks for numerical simulations and MATLAB scripts (R2020a Update 4) for figure generation are available at: https://github.com/dbli2000/SARS-CoV2Bloom-Filter.

For manuscripts utilizing custom algorithms or software that are central to the research but not yet described in published literature, software must be made available to editors and

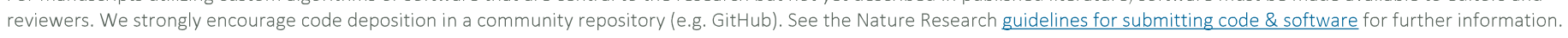

\section{Data}

Policy information about availability of data

All manuscripts must include a data availability statement. This statement should provide the following information, where applicable:

- Accession codes, unique identifiers, or web links for publicly available datasets

- A list of figures that have associated raw data

- A description of any restrictions on data availability

SARS-CoV-2 genomic sequences are available at NCBI (https://www.ncbi.nlm.nih.gov/sars-cov-2/). Read statistics and read counts are provided in Supplementary Data 2. Example LAMP-Seq data are available at SRA (accession number PRJNA729981). 
Please select the one below that is the best fit for your research. If you are not sure, read the appropriate sections before making your selection.

$\bigotimes$ Life sciences $\quad \square$ Behavioural \& social sciences $\quad \square$ Ecological, evolutionary \& environmental sciences

For a reference copy of the document with all sections, see nature.com/documents/nr-reporting-summary-flat.pdf

\section{Life sciences study design}

All studies must disclose on these points even when the disclosure is negative.

Sample size For each investigation involving human research participants, the sample size was determined by the maximum number of available research participants or clinical samples.

Data exclusions Clinical samples (panel 3D) were left-over specimen from routine clinical diagnostics. Individual samples were excluded if they did not meet quality criteria (i.e., failure of internal extraction control). For each analyzed sample, all replicates were included in data analysis.

Replication The number of replicates are indicated for each Figure panel. No contradictory results were obtained.

Randomization In our study, no assignment to groups took place. Therefore, no randomization was required.

Blinding $\quad$ Test results obtained from LAMP-Seq (positive / negative) and from qPCR (Ct-values) were generated by the data analysis algorithm (LAMPSeq, see Methods) and the LightCycler software, respectively. Therefore, no blinding was required. Clinical samples were pseudonymized.

\section{Reporting for specific materials, systems and methods}

We require information from authors about some types of materials, experimental systems and methods used in many studies. Here, indicate whether each material, system or method listed is relevant to your study. If you are not sure if a list item applies to your research, read the appropriate section before selecting a response.

\begin{tabular}{|c|c|}
\hline$n / a$ & Involved in the study \\
\hline Х & $\square$ Antibodies \\
\hline & $\bigotimes$ Eukaryotic cell lines \\
\hline Х & Palaeontology and archaeology \\
\hline$\bigotimes$ & $\square$ Animals and other organisms \\
\hline$\square$ & $\bigotimes$ Human research participants \\
\hline Х & Clinical data \\
\hline Х & $\square$ Dual use research of concern \\
\hline
\end{tabular}

Methods

n/a Involved in the study

$\bigotimes \square$ ChIP-seq

Х $\square$ Flow cytometry

\ $\square$ MRI-based neuroimaging

Eukaryotic cell lines

Policy information about cell lines

Cell line source(s)

ATCC CRL-1586 (VERO-E6), ATCC CCL-81 (VERO) were purchased from ATCC.

Authentication

For the two cell lines used in the study, no approved authentication protocol exist. Therefore we purchased the cell lines from an official supplier (ATCC), confirmed their origin (monkey) using NGS, and confirmed that they follow published characteristics as described by ATCC, including shape, growth rate and sensitivity to viral infections.

Mycoplasma contamination

All cell lines were tested negative for mycoplasma.

Commonly misidentified lines

(See ICLAC register)

No commonly misidentified cell lines were used in the study.

\section{Human research participants}

Policy information about studies involving human research participants

Population characteristics

Recruitment
No population-specific characteristics were assessed at any point.

Recruitment of human research participants was either performed upon direct contact after individuals tested positive at the University Hospital Bonn, or were invited to participate in the UKB pilot study through public announcements. These specimens were collected upon informed consent under a human subjects protocol approved by the ethics committee of the 
Medical Faculty of the University Hospital Bonn, Germany (149/20 and 500/20). No data on age, gender or ethnicity were collected. Additional viral samples were obtained from diagnostic laboratories as left-over specimen. Clinical samples were enriched for a previous positive qPCR result, thus increasing the prevalence of positives in our clinical cohorts. No selfselection took place. University Hospital Bonn, under accession numbers $149 / 20$ and 500/20.

Note that full information on the approval of the study protocol must also be provided in the manuscript. 